
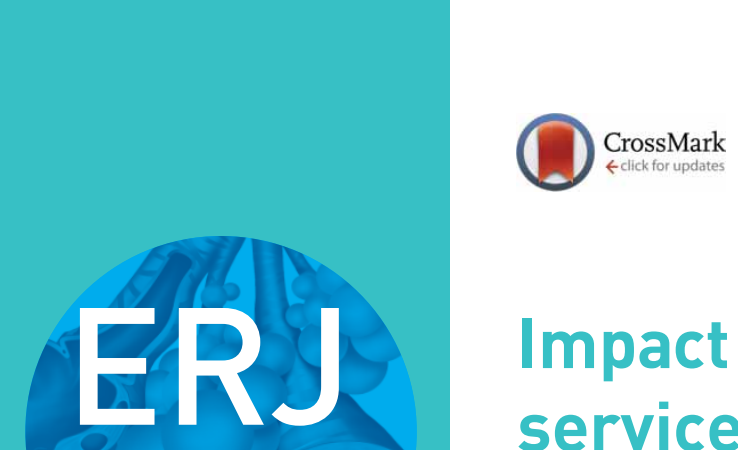

open

\title{
Impact of a protocol-driven unified service for neonates with bronchopulmonary dysplasia
}

\author{
Natalie Batey (i] ${ }^{1}$ Dushyant Batra ${ }^{1}$, Jon Dorling ${ }^{1,2}$ and Jayesh Mahendra Bhatt $\mathbb{B}^{3}$
}

Affiliations: ${ }^{1}$ Nottingham Neonatal Service, Nottingham University Hospitals NHS Trust, Nottingham, UK. ${ }^{2}$ Dept of Academic Child Health, University of Nottingham, Nottingham, UK. ${ }^{3}$ Paediatric Respiratory Dept, Nottingham Children's Hospital, Nottingham University Hospitals NHS Trust, Nottingham, UK.

Correspondence: Jayesh M. Bhatt, Nottingham Children's Hospital, Queens Medical Centre, Nottingham NG7 2UH, UK. E-mail: jayesh.bhatt@nuh.nhs.uk

\section{ABSTRACT}

Aim: A new specialised service for preterm infants with bronchopulmonary dysplasia requiring long-term oxygen therapy (LTOT) was established in 2007, led by the paediatric respiratory team, transitioning from neonatal-led follow-up. The new service included the utilisation of a clear protocol. Our objective was to review whether this service initiation led to a reduction of time in LTOT and hospital readmissions.

Methods: We performed a retrospective cohort study of infants born at <32 weeks' gestation requiring LTOT in a single tertiary neonatal service. Cases were identified from hospital records, BadgerNet and a local database for two cohorts, 2004-2006 and 2008-2010. Data collected for infants requiring LTOT included demographic details, length of neonatal stay, time in oxygen and hospital attendance rates.

Results: The initiation of the service led to an increase in the number of discharges in LTOT: $13.1 \%$ of infants born alive before 32 weeks' gestation in comparison to $3.5 \%(p<0.001)$. However, the length of time in LTOT reduced from 15 to 5 months $(\mathrm{p}=0.01)$. There was no difference in hospital readmission rates $(\mathrm{p}=0.365)$.

Conclusions: In our experience the increase in neonates requiring LTOT is likely to be due to enhanced provision of overnight oximetry studies prior to discharge. Structured monitoring and weaning led to a shorter duration of home oxygen therapy.

@ERSpublications

The implementation of a clear protocol for assessment and management of neonates with bronchopulmonary dysplasia may lead to more infants being discharged in home oxygen, but has potential to reduce overall healthcare costs and improve long-term outcomes http://ow.ly/fYcv30nIc2c

Cite this article as: Batey N, Batra D, Dorling J, et al. Impact of a protocol-driven unified service for neonates with bronchopulmonary dysplasia. ERJ Open Res 2019; 5: 00183-2018 [https://doi.org/ 10.1183/23120541.00183-2018].

Received: Oct 142018 | Accepted after revision: Jan 282019

Copyright $\odot$ ERS 2019. This article is open access and distributed under the terms of the Creative Commons Attribution Non-Commercial Licence 4.0. 


\section{Introduction}

Bronchopulmonary dysplasia (BPD) is the leading cause of chronic lung disease in infancy [1] and the main reason for long-term oxygen therapy (LTOT) in children [2]. Ongoing respiratory health problems and abnormal lung function can persist into childhood and adolescence [3-5]. BPD is defined as the need for oxygen 28 days after birth and an oxygen requirement at 36 weeks post-conceptual age [1]. Severity is graded according to oxygen requirements at 36 weeks corrected gestational age [6].

LTOT is recommended by the British Thoracic Society (BTS) for neonates with BPD to reduce or prevent pulmonary hypertension, reduce intermittent desaturations, reduce airway resistance, promote growth and provide neurodevelopmental benefit [7]. It may reduce the risk of sudden infant death and neurodevelopmental impairment [7].

In our tertiary neonatal service there was a change in care for infants with BPD being discharged in LTOT. Historically, the neonatal team provided individual consultant-led outpatient care. In 2007, a new respiratory paediatric team was developed comprising a tertiary paediatric respiratory consultant and specialist community nursing team.

Prior to the initiation of the new service, discharge home in LTOT, follow-up and review of overnight oximetry studies was at the discretion of the named neonatologist. There was no formal guidance for the review of overnight oximetry studies or oxygen weaning, either as an inpatient or outpatient.

Our hypothesis was that a formalised protocol-led service for BPD would reduce both the length of time infants spent in LTOT and the number of hospital readmissions.

\section{Methods}

\section{Study population}

We performed a retrospective cohort study of neonates with BPD requiring LTOT. Two cohorts admitted to our tertiary neonatal service were identified. As the service transitioned in 2007, we chose the two cohorts 2004-2006 and 2008-2010. The cohorts were kept close to minimise the effects of any other change in management or policy affecting length of stay or clinical outcomes.

\section{Study design}

Cases were identified using two methods. All admissions at <32 weeks' gestation in the 2004-2006 cohort were identified from admission records and the hospital patient administration system, and their discharge letters reviewed. For any infants discharged in oxygen or where oxygen status was unclear, further data were gathered from the medical notes. Details of the 2008-2010 cohort were obtained from a locally kept database detailing all infants discharged from the neonatal units in oxygen. We excluded any infants followed-up for their home oxygen in other centres or born at $>32$ weeks' gestation.

The number of live births was obtained from hospital records. The mother's postcode was reviewed in order to ensure inclusion of only those infants resident in the geographical area followed-up by the respiratory team. Data on demographic details and length of neonatal stay were obtained from BadgerNet (a UK-based paperless neonatal record; www.clevermed.com/badgernet/badgernet-neonatal/), or where this was not available, from hospital notes. Data on the length of time in oxygen were extracted from patient records for the first cohort and the local database for the second.

Admissions after neonatal discharge were identified from both hospital systems and patient notes. Data were extracted on the number of admissions in the first year after discharge from the neonatal unit, the length of these admissions, whether they were respiratory in origin or not and the number of emergency department attendances without admission.

\section{Protocol}

After transition of the service, an overnight pulse oximetry study was recommended for all infants in $\geqslant 0.1 \mathrm{~L} \cdot \mathrm{min}^{-1}$ oxygen at 36 weeks. Infants in $<0.1 \mathrm{~L} \cdot \mathrm{min}^{-1}$ oxygen are reviewed at 38 weeks: if still in oxygen, overnight oximetry is performed in $0.1 \mathrm{~L} \cdot \mathrm{min}^{-1}$ oxygen; if out of oxygen, the study is performed in air. The respiratory team meets the family, and performs and analyses the overnight oximetry studies, which are recorded for a minimum of 6-8 h. During the study a diary is kept of feeds and any other interventions and is used when analysing the oximetry data. The result of the study guides the home oxygen provision. Oximetry targets in LTOT are [7] average arterial oxygen saturation measured by pulse oximetry $\left(\mathrm{SpO}_{2}\right)$ of $\geqslant 93 \%$ ( $\geqslant 95 \%$ if evidence of pulmonary hypertension); $\mathrm{SpO}_{2}>90 \%$ for $>95 \%$ of artefact-free total study time; and fluctuations in oxygen saturations from the baseline (the number of times per hour where the oxygen saturation falls by $\geqslant 4 \%$ from the baseline) should be less than four per hour. Infants requiring LTOT are followed-up in the community and in clinic to coordinate further overnight oximetry studies, oxygen weaning and palivizumab immunisation during winter months. An 
overnight pulse oximetry study is performed within $48 \mathrm{~h}$ of neonatal discharge, then at 3-5-week intervals until in $0.1 \mathrm{~L} \cdot \mathrm{min}^{-1}$ oxygen. A 2-h daytime study (while awake and feeding) is then performed; if the baby is able to maintain $\mathrm{S}_{\mathrm{PO}_{2}} \geqslant 93 \%$, the parents are advised to give the baby time in air during the day. The time is gradually increased by an hour a day until the baby is off oxygen in the daytime while awake. Next, an overnight oximetry study takes place in air. If passed, the infant goes into air and has a further overnight study 3 months later. Oxygen equipment is removed from the home once the infant has either coped with at least one viral upper respiratory tract infection or after the end of winter.

Infants are seen in the respiratory clinic 4-6 weeks after neonatal discharge then every 3 months for at least the first year; this is adjusted if there are concerns, including those regarding oximetry results, respiratory infections, hospital admissions or growth. During the winter months, infants are reviewed at a monthly palivizumab clinic. After 1 year children are seen at least every 6 months and only discharged back to their general practitioner if asymptomatic. Home oxygen saturation monitors are not routinely provided and parents are advised to contact the community nursing team or attend the emergency department if they are at worried about any change in their child. All stages of management have a clear protocol as per the published BTS guidelines [7].

\section{Statistical analyses}

All data were nonparametric, so are presented as median (range). Statistical analyses were performed using SPSS version 21 (IBM, Armonk, NY, USA). Mann-Whitney U-tests and Fisher's exact tests were used to test the significance of continuous data, and Chi-squared tests were used for categorical data. The chair of the local ethics committee agreed that full ethical approval was not required.

\section{Results}

There were 451 live births of infants at <32 weeks' gestation during 2004-2006 and 337 during 2008-2010 with postcodes within the geographical area followed-up by the respiratory team. Prior to the initiation of a respiratory service, $3.5 \%$ of infants born at $<32$ weeks' gestation were discharged in home oxygen; afterwards this rose to $13.1 \%(\mathrm{p}<0.001)$.

There were no differences in the demographic details between the two groups (table 1). The median (range) length of neonatal admission was 119 (85-271) days in 2004-2006 and 110 (40-233) days in 2008-2010 ( $\mathrm{p}=0.134)$. There was at least one hospital admission in $12(75.0 \%)$ infants born between 2004 and 2006 and 29 (65.9\%) of the infants born between 2008 and 2010 ( $p=0.753)$. Eight (50.0\%) infants in the 2004-2006 cohort and $24(54.5 \%)$ of the 2008-2010 cohort ( $p=0.935)$ required admission for a respiratory cause. One infant died during a readmission in the 2004-2006 cohort; they were excluded from subsequent analyses.

There was a significant reduction in the length of time infants spent in LTOT (table 2). However, there was no difference in the number of hospital admissions (total and due to respiratory cause) or time spent in hospital during respiratory admissions. There was an increase in the number of emergency department attendances in the second cohort $(\mathrm{p}=0.05)$.

The summary statistics for the last oximetry study prior to coming off oxygen for the second cohort are shown in table 3.

\section{Discussion}

Our study has shown that integration of a protocol-driven specialist service with community support for neonates with BPD being discharged in LTOT is associated with a significant increase in the number of infants discharged in LTOT, but also a significantly reduced length of time in LTOT.

\section{TABLE 1 Comparison of demographic details of the two cohorts, 2004-2006 and 2008-2010}

\begin{tabular}{|c|c|c|c|}
\hline & 2004-2006 & 2008-2010 & p-value \\
\hline Subjects & 16 & 44 & \\
\hline Birthweight g & $720(480-1000)$ & 755 (410-1300) & $0.564^{\#}$ \\
\hline Gestation weeks & $25(23-29)$ & $26(23-29)$ & $0.358^{\#}$ \\
\hline Male & $10(62.5)$ & $23(52.3)$ & $0.481^{9}$ \\
\hline
\end{tabular}


TABLE 2 Comparison of outcomes between the two cohorts, 2004-2006 and 2008-2010

\begin{tabular}{lccc} 
& $\mathbf{2 0 0 4 - 2 0 0 6}$ & $\mathbf{2 0 0 8 - 2 0 1 0}$ & p-value \\
\hline Time in oxygen months & $15(0-66)$ & $5(2-24)$ & 0.01 \\
Total hospital readmissions & $1(0-17)$ & $1(0-5)$ & 0.365 \\
Total respiratory-related readmissions & $1(0-8)$ & $1(0-5)$ & 0.948 \\
Length of respiratory admissions days & $4.5(0-263)$ & $5(0-105)$ & 0.717 \\
Total emergency department attendances & $0(0-1)$ & $1(0-6)$ & 0.05 \\
& & \\
\hline
\end{tabular}

That $13.1 \%$ of live births at $<32$ weeks' gestation in the later cohort were discharged in LTOT is similar to quoted percentages in the literature, ranging from $8.9 \%$ to $17 \%$ of live births at $<32$ weeks' gestation [8-10]. The proportion increases with decreasing gestational age, with $36-43 \%$ of infants live-born at $<26$ weeks' gestation being discharged in LTOT $[3,11]$. One study found that improved survival in very premature infants at the highest risk of BPD (oxygen requirement at 36 weeks) has led to a significant increase in BPD rates, with a U-shaped distribution over time [12]. However, this is not evidenced in other studies [13]. There does appear to be significant variation between centres in the percentage of extremely and very preterm infants discharged in LTOT. An American multicentre study of 8167 infants born before 32 weeks' gestation found a four- to five-fold institutional variation in discharge in LTOT of babies with BPD, with the incidence ranging between $7 \%$ and $95 \%$ [8]. A UK study of four centres found that $8-64 \%$ of infants with BPD were discharged with LTOT, despite similar proportions of chronic lung disease diagnoses [14].

The increase in the number of infants discharged in LTOT in our service is likely to be secondary to increased awareness and the introduction of a local guideline based upon BTS guidance [7]. Increased awareness has meant that more infants undergo overnight oximetry studies, including those with less severe BPD in air. Those with abnormal results are discharged in LTOT. It is likely that in the first cohort, babies with mild or moderate BPD may have been weaned from oxygen rather than having overnight sleep oximetry. Therefore, only those with severe BPD were discharged in LTOT. This was not based on a formal physiological assessment of oxygen need. The retrospective nature of data collection does not allow us to evidence this. There was a nonsignificant difference in the length of stay on the neonatal unit. We speculate that this may indicate earlier discharge in the absence of a formal, protocol-driven assessment of oxygen need in the first cohort. Alternatively, the nonsignificant difference may be due to the fact the two cohorts are temporally close together with only one year separating the two. It is likely that changes in practice take time to filter through; now that this protocol is well embedded in practice, it seems that babies are being discharged home earlier, although we have not formally analysed this.

We acknowledge that for each cohort, knowing the length of time in oxygen of all babies born $<32$ weeks' gestation who were still in oxygen at 36 weeks, regardless of whether they came out of oxygen at home or in hospital would allow a more robust analysis. However, we are confident that the length of home oxygen therapy in the later cohort is related to this new service model; current analysis shows that the median duration of home oxygen therapy has continued to reduce (data not shown).

Home oxygen can lead to an earlier discharge, which is beneficial in terms of healthcare economics, as it reduces inpatient days on the neonatal unit $[7,14]$. A study of four UK neonatal services found that those with a high use of LTOT ( $>50 \%$ of infants with BPD) had shorter neonatal unit stays and similar numbers

\section{TABLE 3 Statistics of last oximetry study prior to discontinuing oxygen}

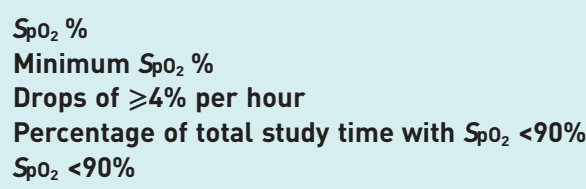

Data are presented as median (interquartile range), unless otherwise stated. $\mathrm{SpO}_{2}$ : arterial oxygen saturation measured by pulse oximetry. 
of hospital readmissions compared to those with low oxygen LTOT rates $(<20 \%$ of infants with BPD) [14]. Although this led to a longer period of home LTOT, overall costs of care for the first 2 years of life were significantly lower in centres with high use of LTOT [14]. In addition, there are benefits to the family of early discharge, as bonding between parents and their children is improved in the home environment [15]. Other benefits may include less feeding difficulty, improved growth and improved neurodevelopmental outcomes [7].

Our service has a structured weaning programme based upon BTS guidance [7, 16]. Prior to these guidelines there was no set recommendation on the performance of oximetry studies and use was as advised by the named neonatologist. We believe that the new structured approach is the reason for the reduction in time in home oxygen and ensures that oxygen therapy is stopped appropriately, neither too late nor too early. However, due to the increase in the number of infants discharged in LTOT, there is likely to be a number of infants with milder BPD who will stop their LTOT earlier. The median duration of home oxygen therapy in our later cohort is comparable to or shorter than published data from other studies (table 4).

LTOT is supported in the community by respiratory specialist nurses, who also help provide holistic care for these families [15]. A reduction in the length of time in oxygen also benefits the family by reducing the burden of social isolation. In addition, it removes the prolonged need for transporting equipment and ensuring adequate quantities of oxygen in cylinders whenever families wish to leave the home.

Children with BPD are at increased risk of respiratory tract infections $[10,11]$. Our study did not show a difference in the number of readmissions between the two cohorts. It has been shown that infants in LTOT are more likely to require hospital admission [10, 11, 23, 24]. GREENOUGH et al. [24] demonstrated that until the age of 2 years, infants born at $<32$ weeks' gestation with BPD discharged in LTOT were significantly more likely to be admitted to hospital and attend as outpatients than infants with BPD not requiring LTOT. There was no difference in the number of paediatric intensive care unit admissions or length of stay. The authors did not differentiate between respiratory and nonrespiratory admissions, but a later study reported no difference in the number of respiratory syncytial virus (RSV)-related admissions [25]. Hong et al. [10] found that infants on LTOT with BPD had both significantly more respiratory admissions overall and significantly more RSV admissions within the first 3 years of life. GreENOUGH and co-workers $[25,26]$ found that the significant difference in hospital admissions disappeared after the age of 2 years, but outpatient attendances, both overall and for respiratory care, were significantly higher at ages 2-7 years. In our service, early contact with the respiratory team before discharge ensures that these complex patients are known to, and can be cared for, by a team of health professionals if they are readmitted to paediatric services.

It has been shown that poor respiratory health and impaired lung function in infants with BPD extends into childhood and adolescence $[1,4,5,27,28]$. Children who have suffered BPD are more likely to develop episodes of wheezing $[27,29,30]$. The respiratory team follow-up children in the outpatient setting until they no longer require LTOT and have had two winters without severe, frequent or persistent respiratory symptoms [16]. This follow-up ensures that ongoing or persistent respiratory symptoms are detected and can be treated. In services where BPD and LTOT are managed by clinicians other than respiratory specialists, referral to a paediatric respiratory team for infants who are having multiple respiratory exacerbations may be prudent. Being under respiratory follow-up and the fact that parents are advised to contact the community nursing team if they are worried about any change in clinical status is

\section{TABLE 4 Comparison of duration of home oxygen therapy}

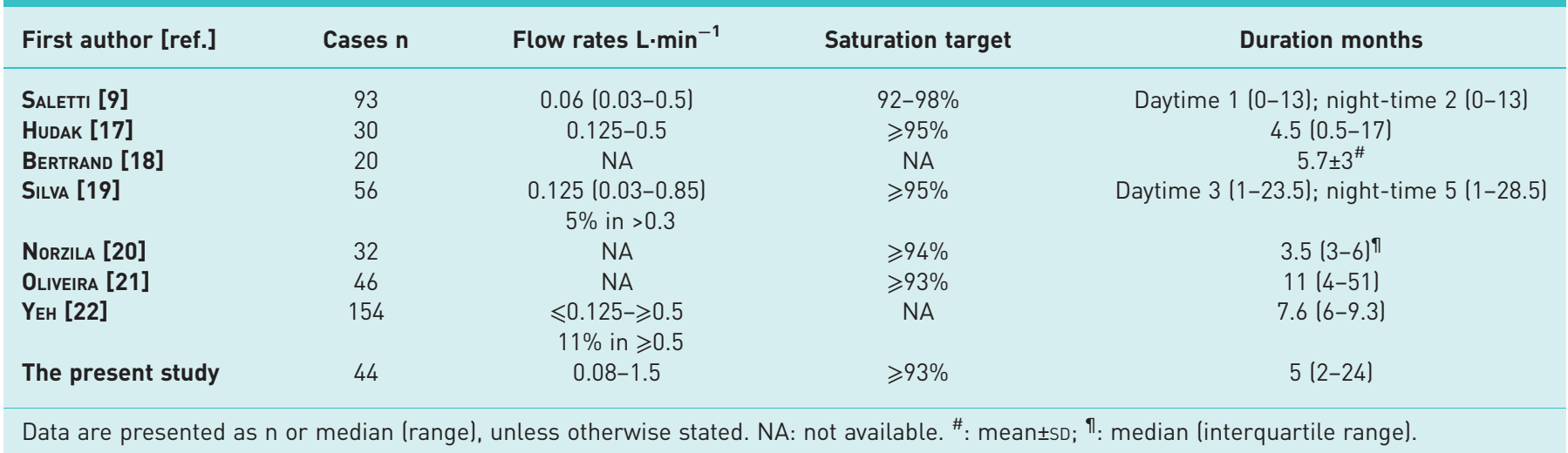


likely to have altered the threshold for admission in these babies, because they are perceived to be more vulnerable. Review and reassurance by the respiratory team then leads to a short hospital stay at readmission.

There are limitations to our study, the first being the small sample numbers. It is a study based on data from a single UK National Health Service trust. During the period of the study there was a transition from two-site paediatric services to a single children's hospital. During this transition, attendance policy altered. Prior to single-site care, infants with complex needs had direct ward access, but following the merger they were required to present via their community nurse, general practitioner or the emergency department. This may account for the change in the number of emergency department attendances. We did not have data on admissions elsewhere, so there may have been uncounted admissions and emergency department attendances at other hospitals.

Being a retrospective cohort study has limited the data we have been able to collect. For the duration in oxygen we were reliant upon what was recorded in the clinical notes, so in some cases lack of precision on the number of days in home oxygen in the 2004-2006 cohort meant we had to present data in months rather than days, which would have improved the accuracy of our results. Although we made every effort to identify infants being discharged in oxygen, due to the nature of the retrospective study we may not have identified some cases from the first cohort.

Although there was no statistical difference in the length of neonatal admission, we do not have data on the gestational age at which every infant born at $<32$ weeks' gestation during both periods stopped oxygen therapy. It is likely the increased awareness of the new guidelines and protocol meant that more infants underwent overnight oximetry studies and so were more likely to be discharged home in oxygen. This increased awareness coincided with new national guidelines, a new service model and the involvement of new clinicians.

Future work should include longitudinal reviews of duration in oxygen and length of neonatal admission. This should include data collection on other outcomes and reviews of discharge policies. Local cost evaluations should be performed to assess whether there are savings from the reduction of time in oxygen despite the increase in patients discharged in LTOT. Little research is available on family perceptions of home oxygen and data in this area would help guide the management and support from the multidisciplinary team. In view of the national and international variation in the use of LTOT, a widespread study of current practices for discharge assessment and follow-up structure would be prudent.

Although a structured discharge policy and specialised service led to an increase in the number of infants discharged in oxygen, it has standardised clinical care and has the potential to reduce overall healthcare costs by reducing the length of neonatal admissions.

Conflict of interest: N. Batey has nothing to disclose. D. Batra reports a conference and travel bursary to attend the Joint European Neonatal Societies conference in 2017 from the British Association of Perinatal Medicine which was funded by Chiesi Limited. J. Dorling has nothing to disclose. J.M. Bhatt has nothing to disclose.

\section{References}

Baraldi E, Filippone M. Chronic lung disease after premature birth. N Engl J Med 2007; 357: 1946-1955.

2 Primhak RA, Hicks B, Shaw NJ, et al. Use of home oxygen for children in England and Wales. Arch Dis Child 2011; 96: 389-392.

3 Hennessy EM, Bracewell MA, Wood N, et al. Respiratory health in pre-school and school age children following extremely preterm birth. Arch Dis Child 2008; 93: 1037-1043.

4 Doyle LW, Faber B, Callanan C, et al. Bronchopulmonary dysplasia in very low birth weight subjects and lung function in late adolescence. Pediatrics 2006; 118: 108-113.

5 Doyle LW, Anderson P, Callanan C, et al. Respiratory function at 8-9 years in in extremely low birth weight/very preterm children born in Victoria 1991-1992. Pediatr Pulmonol 2006; 41: 570-576.

6 Jobe AH, Bancalari E. Bronchopulmonary dysplasia. Am J Respir Crit Care Med 2001; 163: 1723-1729.

7 Balfour-Lynn IM, Field DJ, Gringras P, et al. BTS guidelines for home oxygen in children. Thorax 2009; 64: Suppl. 2, iil-ii26.

8 Lagatta J, Clark R, Spitzer A. Clinical predictors and institutional variation of home oxygen use in preterm infants. J Pediatr 2012; 160: 232-238.

9 Saletti A, Stick S, Doherty D, et al. Home oxygen therapy after preterm birth in Western Australia. J Paediatr Child Health 2004; 40: 519-523.

10 Hong T, Bolisetty S, Bajuk B, et al. A population study of respiratory rehospitalisation in very preterm infants in the first 3 years of life. J Paediatr Child Health 2016; 52: 715-721.

11 Luu TM, Lefebvre F, Riley P, et al. Continuing utilisation of specialised health services in extremely preterm infants. Arch Dis Child Fetal Neonatal Ed 2010; 95: F320-F325.

12 Chen F, Bajwa NM, Rimensberger PC, et al. Thirteen-year mortality and morbidity in preterm infants in Switzerland. Arch Dis Child Fetal Neonatal Ed 2016; 101: F377-F383.

13 Costeloe KL, Hennessy EM, Haider S, et al. Short term outcomes after extreme preterm birth in England: comparison of two birth cohorts in 1995 and 2006 (the EPICure studies). BMJ 2012; 345: e7976. 
14 Greenough A, Alexander J, Burgess S, et al. High versus restricted use of home oxygen therapy, health care utilisation and the cost of care in chronic lung disease infants. Eur J Pediatr 2004; 163: 292-296.

15 Kotecha S, Allen J. Oxygen therapy for infants with chronic lung disease. Arch Dis Child Fetal Neonatal Ed 2002; 87: F11-F14.

16 Khetan R, Hurley M, Spencer S, et al. Bronchopulmonary dysplasia within and beyond the neonatal unit. $A d v$ Neonatal Care 2016; 16: 17-25.

17 Hudak BB, Allen MC, Hudak ML, et al. Home oxygen therapy for chronic lung disease in extremely low-birth-weight infants. Am J Dis Child 1989; 143: 357-360.

18 Bertrand P, Alvarez C, Fabres J, et al. Oxigenoterapia domiciliaria en niños con displasia broncopulmonar. [Home oxygen therapy in children with chronic respiratory failure]. Rev Med Chil 1998; 126: 284-292.

19 Silva DT, Hagan R, Sly PD. Home oxygen management of neonatal chronic lung disease in Western Australia. J Paediatr Child Health 1995; 31: 185-188.

20 Norzila MZ, Azizi BH, Norrashidah AW, et al. Home oxygen therapy for children with chronic lung diseases. Med J Malaysia 2001; 56: 151-157.

21 Oliveira L, Coelho J, Ferreira R, et al. Oxigenoterapia Domiciliária de Longa Duração na Criança: Evidências e Questões em Aberto. [Long-term home oxygen therapy in children: evidences and open issues]. Acta Med Port 2014; 27: 717-725.

22 Yeh J, McGrath-Morrow SA, Collaco JM. Oxygen weaning after hospital discharge in children with bronchopulmonary dysplasia. Pediatr Pulmonol 2016; 51: 1206-1211.

23 Groothuis JR, Gutuerrez KM, Lauer BA. Respiratory syncytial virus infection in children with bronchopulmonary dysplasia. Pediatrics 1988; 82: 199-203.

24 Greenough A, Alexander J, Burgess S, et al. Home oxygen status and rehospitalisation and primary care requirements of infants with chronic lung disease. Arch Dis Child 2002; 86: 40-43.

25 Greenough A, Alexander J, Boorman J, et al. Respiratory morbidity, healthcare utilisation and cost of care at school age related to home oxygen status. Eur J Pediatr 2011; 170: 969-975.

26 Greenough A, Alexander J, Burgess S, et al. Preschool healthcare utilisation related to home oxygen status. Arch Dis Child Fetal Neonatal Ed 2006; 91: F337-F341.

27 Fawke J, Lum S, Kirkby J, et al. Lung function and respiratory symptoms at 11 years in children born extremely preterm: the EPICure study. Am J Respir Crit Care Med 2010; 182: 237-245.

28 Ronkainen E, Dunder T, Peltoniemi O, et al. New BPD predicts lung function at school age: follow-up study and meta-analysis. Pediatr Pulmonol 2015; 50: 1090-1098.

29 Northway WH, Moss RB, Carlisle KB, et al. Late pulmonary sequelae of bronchopulmonary dysplasia. $N$ Engl J Med 1990; 323: 1793-1799.

30 Doyle LW, Anderson PJ. Long-term outcomes of bronchopulmonary dysplasia. Semin Fetal Neonatal Med 2009; 14: $391-395$. 